\title{
Review
}

\section{T-cell death and cancer immune tolerance}

\author{
B Lu ${ }^{\star, 1,2}$ and OJ Finn ${ }^{1,2}$
}

\begin{abstract}
Cancer patients mount adaptive immune responses against their tumors. However, tumor develops many mechanisms to evade effective immunosurveillance. T-cell death caused by tumor plays a critical role in establishing tumor immunotolerance. Chronic stimulation of $T$ cells by tumors leads to activation-induced cell death. Abortive stimulation of $T$ cells by tolerogenic antigenpresenting cells loaded with tumor antigens leads to autonomous death of tumor-specific T cells. Therapeutic approaches that prevent T-cell death in the tumor microenvironment and tumor draining lymph nodes, therefore, should boost adaptive immune responses against cancer.
\end{abstract}

Cell Death and Differentiation (2008) 15, 70-79; doi:10.1038/sj.cdd.4402274; published online 16 November 2007

Paul Ehrlich theorized that the immune system is critical in preventing the outgrowth of 'overwhelming frequency' of cancers. He proposed that both native and adaptive immune defenses play a role. ${ }^{1}$ Indeed, in mice, syngenic transplant tumors could be shown to elicit specific and protective immune responses against 'tumor-specific antigens'. ${ }^{2}$ Based on these findings, Macfarlane Burnet extended Paul Ehrlich's theory and coined the word 'immunosurveillance' to describe the concept of an immunological resistance against cancer development. ${ }^{3,4}$ It is believed that both innate and adaptive immune responses are important in recognizing and eliminating cancer cells. ${ }^{3,4}$ We now understand that cancer development is a result of accumulation of somatic mutations and dysregulated oncogenes and tumor suppressor genes. These genetic abnormalities often create altered self-antigens in cancer cells, many of which have already been shown to be recognized by the immune system. ${ }^{5}$

Many recent studies provided strong support for the existence of immunosurveillance. IFN $\gamma$ neutralization with a specific monoclonal antibody abrogated the LPS-induced rejection of a fibrosarcoma cell line Meth $A$ in BALB/c mice. ${ }^{6}$ Besides transplanted tumor models, the antitumor activity of IFN $\gamma$ was also observed in carcinogen-induced and spontaneous tumor models. Compared to wild-type mice, IFN $\gamma$ deficient mice and Stat-1-deficient mice developed carcinogen methylcholanthrene-induced tumors at faster kinetics and higher frequencies. ${ }^{7}$ IFN $\gamma$-insensitive p53-deficient mice developed a broader range of tumors compared with mice lacking p53 alone. $^{7}$ IFN $\gamma$-deficient mice on the C57BL/6 background developed disseminated lymphomas. ${ }^{8}$ Some of the effects of IFN $\gamma$ are mediated by the innate immune system, particularly NK T cells ${ }^{8}$ and $\gamma \delta$ T cells. ${ }^{9,10}$ IFN $\gamma$ is the hallmark cytokine for Th1 cells and $\mathrm{CD}^{+}{ }^{+} \mathrm{T}$ cells and is critical for cell-mediated immunity and cell-mediated adaptive immune responses which are important for immunosurveillance. $^{11}$

In humans, the presence of certain subsets of lymphocytes within the tumor can be a favorable prognostic sign. For example, the presence of intratumoral $\mathrm{T}$ cells correlates with better clinical outcome in advanced colorectal carcinoma. ${ }^{12} \mathrm{~A}$ higher density of memory T-cell markers within a tumor is favorably associated with overall survival of colorectal cancer patients. ${ }^{13}$ Collectively, these data support the immunosurveillance hypothesis.

Despite the presence of immunosurveillance, cancers can develop in apparently immunocompetent animals and humans. Avoidance of immunosurveillance is proposed to be the seventh hallmark of cancer. ${ }^{14,15}$ This happens through many well-characterized mechanisms including induction of T-cell tolerance by autochthonous tumors, ${ }^{16}$ cancer immunoediting, ${ }^{17}$ and development of an immune suppressive cancer microenvironment. ${ }^{18}$ Therapeutic cancer vaccines or adoptive immunotherapy are being developed and tested as potential approaches to strengthen the immune responses after tumor arise in order to slow their progression and prevent their recurrence. So far immunotherapeutic approaches have been only partially successful. ${ }^{19}$ It remains important, therefore, to better understand how tumors manage to outmaneuver immune recognition and elimination in the first place, and how they ultimately progress to widely disseminated cancer. In this review, we focus on how tumors evade effective immunosurveillance by causing T-cell death.

\footnotetext{
${ }^{1}$ Department of Immunology, School of Medicine, University of Pittsburgh, Pittsburgh, PA, USA and ${ }^{2}$ University of Pittsburgh Cancer Institute, Pittsburgh, PA, USA *Corresponding author: B Lu, Department of Immunology, School of Medicine, University of Pittsburgh, 200 Lothrop Street, Pittsburgh, PA 15261, USA.

Tel: + 1412648 9339; Fax: + 1412648 9339; E-mail: Binfeng@ @itt.edu

Keywords: apoptosis; autophagy; immunosurveillance; T-cell

Abbreviations: ASE I, arginase I; ASE II, arginase II; ALPS, the autoimmune lymphoproliferative syndrome; BH, Bcl-2 homology; DC, dendritic cell; EAE, autoimmune experimental allergic encephalomyelitis; HSV, herpes simplex virus; IDO, indoleamine 2,3-dioxygenase; iNOS, the inducible nitric oxide synthase; Env, envelope glycoproteins; QUIN, 3-hydroxyantranylic acid (3-HAA) and quinolinic acid; qVD-OPh, quinoline-Val-Asp-CH2-difluorophenoxy; SEB, staphylococcal enterotoxin B; TDLN, the tumor draining lymph nodes; TILs, tumor infiltrating lymphocytes; TNF-R, tumor necrosis factor receptor; zVAD-fmk, benzyloxycarbonyl-Val-Ala-Aspfluoromethyl ketone

Received 29.8.07; revised 09.10.07; accepted 09.10.07; Edited by G Kroemer; published online 16.11.07
} 


\section{T-cell Death Pathways}

Depending on their prior experience with antigen, peripheral $T$ cells can be divided into three categories, namely naïve $T$ cells, effector $T$ cells, and memory $T$ cells. Naïve $T$ cells become activated when they encounter antigen-presenting cells loaded with the right antigen. This is followed by increase of antigen-specific T-cell numbers due to elevated proliferation and reduced cell death. During the process of active proliferation, activated $T$ cells differentiate into different types of effector T cells. They are primarily responsible for antigen clearance. A subset of these cells then preserves the ability to quickly respond and eradicate the same antigen that may be encountered in the future, due to further differentiation into memory $\mathrm{T}$ cells.

Apoptotic T-cell death. The number of $T$ cells is tightly regulated by several cell death mechanisms. ${ }^{20}$ Apoptosis has been defined as type I programmed cell death, a term which, in contrast to necrosis, implies active participation of a dying cell in its own death. This process can be initiated in response to a range of intrinsic and extrinsic signals. A number of changes in cellular morphology can help to define apoptosis. In the apoptotic cell, chromosomes condense, the nucleus fragments, cytoplasmic volume decreases, organelles compact, the cell membrane fuses with the endoplasmic reticulum, and the cell finally fragments into numerous 'apoptotic bodies,' which are engulfed by surrounding cells. ${ }^{21}$ These morphologic changes are accompanied by other subcellular indicators of apoptosis, including the exposure of phosphatidylserine on the external surface of the cell membrane and a decrease in mitochondrial transmembrane potential. ${ }^{22}$ Caspase activation is the critical biochemical event during apoptosis and is a hallmark of apoptosis. Caspases cleave vital cellular proteins (e.g. lamin, gelsolin) and proteolytically activate latent enzymes that degrade cellular constituents, such as caspase-activated DNase (CAD), also called DFF40 (DNA fragmentation factor of $40 \mathrm{kDa}) .{ }^{23}$

The regulation of death of activated $\mathrm{T}$ cells and memory $\mathrm{T}$ cells and how it might be influenced by the tumor microenvironment is quite relevant to tumor evasion of immunosurveillance. Two main types of cell death are functionally defined in activated T cells, namely activation induced cell death (AICD) and activated T-cell autonomous death (ACAD) ${ }^{24}$ (Figure 1). AICD describes a phenomenon wherein T-cell hybridomas or thymocytes die by apoptosis following activation through their CD3 molecules. It is now known that the in vitro death of T-cell hybridomas and of activated T cells after artificial stimulation through their TCRs is driven mostly by a subset of tumor necrosis factor receptor (TNF-R) family members that includes Fas, TNF-R1, DR3 (TRAMP, wsl-1, APO-3, LARD), DR4 (TRAIL-R1, APO-2), DR5 (TRAIL-R2, TRICK2, KILLER), and DR6, referred to as 'death receptors'. ${ }^{25}$ Engagement of the T-cell receptor (TCR) on T hybridoma cells or activated normal $T$ cells induces expression of both Fas/APO-1 and its ligand, resulting in autocrine or paracrine activation of apoptosis. Fas activation results in the rapid recruitment of FADD to the cytoplasmic membrane. FADD then brings in pro-caspase 8 forming the death-inducing signaling complex

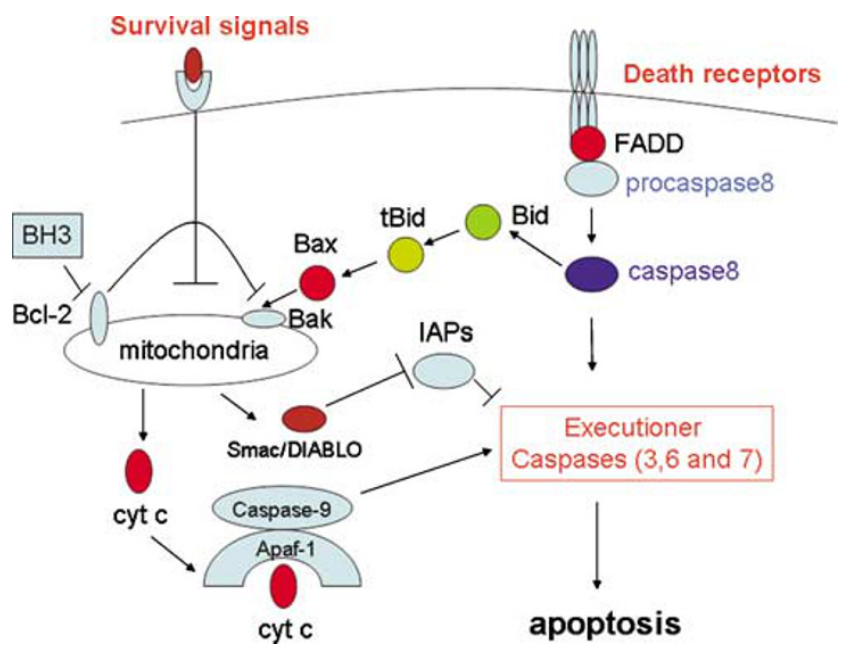

Figure 1 Signaling pathways for AICD and ACAD in T cells. AICD and ACAD are functionally defined cell death mechanisms for activated T cells. AICD is triggered by persistent stimulation of T cells through TCR. In many cases, AICD is mediated by death receptors. ACAD is mainly triggered by the lack of growth factors and cytokines, which provide the survival signal for $T$ cells

(DISC). ${ }^{26}$ Next, proteolytic cleavage of pro-caspase 8 results in its activation and subsequent release from the DISC into the cytoplasm. Depending on the cell context, the downstream signal of caspase 8 is propagated in one of two ways. In so called type I cells, ${ }^{26}$ induction of apoptosis is accompanied by activation of large amounts of caspase 8 by the DISC. Caspase 8 then rapidly cleaves and activates caspase 3 , leading to the effector stage of apoptosis. In type II cells, on the other hand, DISC formation is strongly reduced and a relatively small amount of activated caspase 8 cuts and activates the proapoptotic Bcl-2 family member Bid. ${ }^{26}$ Truncated Bid induces mitochondrial pore formation via Bak or Bax, resulting in the release from mitochondria of proapoptotic molecules such as cytochrome $c$ and Smac/ DIABLO ${ }^{27}$ Cytochrome $c$ can subsequently form a complex with Apaf-1 and pro-caspase 9 in the cytoplasm, to form the apoptosome, which can then activate effector caspases such as caspase 3 and caspase $7 .^{27}$

The Fas pathway functions in vivo to hedge off immune responses against self-antigens. In both human and mice, Fas or FasL deficiency causes the autoimmune lymphoproliferative syndrome (ALPS), a systemic autoimmune disease. ${ }^{28}$ The clinical manifestations of ALPS include massive lymphadenopathy, splenomegaly, autoimmune hemolytic anemia, thrombocytopenia, and other systemic autoimmune symptoms. ${ }^{28}$ In addition, large numbers of CD4 CD8 doublenegative $T$ cells are present in ALPS patients. ALPS patients also have increased numbers of circulating $B$ cells and a polyclonal hypergammaglobulinemia. ${ }^{28}$ Besides systemic autoimmunity, the Fas pathway is also involved in the resolution of organ-specific autoimmunity. Mice showed a delay in their recovery from autoimmune experimental allergic encephalomyelitis (EAE) when the Fas pathway is blocked on T cells. ${ }^{29}$ Given the role of the removal of the Fas/FasL pathway in these autoimmune disorders, there is a possibility that the Fas pathway can be manipulated to enhance immune 
responses to tumor antigens which bear many similarities to self-antigens.

An alternative pathway to death receptors is the phenomenon described as ACAD, ${ }^{24}$ also called cytokine withdrawal cell death and spontaneous T-cell death. ${ }^{25}$ ACAD involves the BCL-2-family-regulated pathway (also known as the intrinsic or mitochondrial pathway) and is responsible for the death of the majority of $T$ cells after cessation of cytokines or growth factors. ${ }^{24,30}$ Members of the Bcl-2 family contain Bcl-2 homology $(\mathrm{BH})$ domains. The prosurvival family members Bcl-2, Bcl-xL, Bcl-w, A1/Bfl-1, and Mcl-1 comprise up to four such homology domains (BH1-4). The Bcl-2 family has two proapoptotic factions. One proapoptotic subgroup possesses three $\mathrm{BH}$ domains (e.g., Bax, Bak (Bcl-2 antagonist/killer), and Bok (Bcl-2-related ovarian killer)). ${ }^{30}$ The other subgroup includes the $\mathrm{BH}$-only proteins Blk (Bik-like killer)/Bik (Bcl-2-like killer)/Nbk, Bid (Bcl-2interacting domain death agonist), Bad (Bcl-2 antagonist of cell death), Harakiri/death protein 5, Noxa/Apr, Bmf (Bcl-2modifying factor), Puma (p53-upregulated modulator of apoptosis)/bbc3, and Bim (Bcl-2-interacting mediator of cell death)/Bod (Bcl-2-related ovarian death gene). All BH3-only proteins induce apoptosis when overexpressed in cultured cells. ${ }^{30} \mathrm{Bcl}-2$-deficient mice developed pleiotropic abnormalities including atrophic thymus and spleen with accelerated apoptotic cell death of lymphocytes. ${ }^{31}$ Overexpression of Bcl-2 does not protect activated T cells from Fas-mediated death; likewise, Fas-deficiency fails to protect $T$ cells from ACAD, which is prevented by Bcl-2 overexpression. ${ }^{24,30}$ Experiments using Bim-deficient mice further confirm the role of $\mathrm{Bcl}-2$ pathway in ACAD. Bim deficiency results in resistance to cytokine withdrawal cell death. When cultured without cytokines (no treatment) or treated with ionomycin, bim-/pre-T cells survived much better than WT cells, similar to what has been observed in Bcl-2-overexpressing $\mathrm{T}$ cells. The bim-I- pre-T cells were, however, as sensitive as the WT cells to treatment with Fas ligand. In addition, the absence of Bim dramatically augmented survival of the resting $T$ cells and markedly protected activated $\mathrm{T}$ cells against cytokine withdrawal. ${ }^{32}$

The Bcl-2 pathway is important for death of activated $T$ cells following acute immune responses. ${ }^{20,33,34}$ Primary evidence was obtained using the staphylococcal enterotoxin $B$ (SEB) challenged mouse model. At the end of the SEBinduced $\mathrm{T}$-cell response, the majority of the activated $\mathrm{V} \beta 8$ $\mathrm{T}$ cells died. Overexpressing $\mathrm{Bcl}-2$ prevented $\mathrm{T}$-cell deletion, but Fas or TNF-R signaling played no role. In addition, $T$ cells from Bim-deficient mice were resistant to SEB-induced deletion. ${ }^{34}$ Further work confirmed the general thesis of this result in acute herpes simplex virus (HSV) infection mouse model. Compared with that in wild-type mice, similar numbers of $\mathrm{HSV}$-specific $\mathrm{CD}^{+} \mathrm{T}$ cells were generated in bim-/- mice during the peak of infection. After the clearance of HSV, the number of HSV-specific CD8 ${ }^{+}$T cells decreased over time in wild-type mice. In contrast, the HSV-specific $\mathrm{CD}^{+} \mathrm{T}$ cells persisted in bim/ mice long after HSV clearance. ${ }^{35}$ Collectively, these studies established that the $\mathrm{Bcl}-2$ pathway mediates T-cell death during the contraction phase of acute T-cell responses.
T-cell Subsets and their Differential Susceptibilities to Cell Death Stimuli. Cell death is regulated differently in subsets of $\mathrm{T}$ cells. Compared to naïve $\mathrm{T}$ cells, effector/ memory $T$ cells express higher levels of death receptors such as Fas and TNF receptors. ${ }^{28}$ The main executioners such as caspase 3 and caspase 6 are also highly expressed in effector $\mathrm{T}$ cells but not expressed in naïve $\mathrm{T}$ cells. ${ }^{36}$ The death receptor pathway is present in activated $T$ cells but not in naïve $\mathrm{T}$ cells. Therefore, the death receptor pathway is associated with TCR signaling and is driven by sustained antigen engagement. Similar to death receptor members, $\mathrm{Bcl}-\mathrm{xl}, \mathrm{Bcl}-\mathrm{a} 1 \mathrm{~b}$, and Bcl-a1d are also highly expressed when TCR is engaged, suggesting their role in protecting antigenengaged $T$ cells against cell death, as these genes are not expressed in resting naïve T cells. ${ }^{36}$ In striking contrast, Bcl2 , which is expressed in high levels in resting naïve and memory $\mathrm{T}$ cells, is downregulated after TCR stimulation. ${ }^{20}$ During T-cell activation, proapoptotic members of the $\mathrm{Bcl} 2$ gene family are regulated not at the transcription levels but at post-translational levels. ${ }^{24}$ The expression pattern of death receptors and $\mathrm{Bcl}-2$ is in agreement that $A I C D$ mediates the TCR-driven death of $T$ cells and ACAD facilitates the nonTCR-driven death of $T$ cells.

In culture, T cells making IFN $\gamma$ such as Th1 cells and CD8 ${ }^{+}$ T cells are intrinsically more prone to apoptosis than Th2 cells which make Th2-type cytokines such as IL-4, IL-5, and $\mathrm{IL}-13 .^{28}$ Expression profiling showed that death receptors and caspase 3 are more highly expressed in Th1 cells than in Th2 cells. ${ }^{36}$ Therefore, hyperactivated Th1 cells are intrinsically self-limiting by apoptotic mechanisms. ${ }^{37,38}$ TCR-induced apoptosis in $\mathrm{CD}^{+}$cells primarily involves Fas, whereas apoptosis in $\mathrm{CD}^{+}{ }^{+} \mathrm{T}$ cells also depends on TNF signaling via the $\mathrm{p} 75$ receptor (TNFR2). ${ }^{28}$

Autophagy and T-cell death. The term 'apoptosis' has long been used as a synonym for programmed cell death. Ultrastructural data suggested the existence of alternative types of programmed cell death, most notably autophagic cell death. ${ }^{39}$ Autophagy is a cellular process by which a portion of the cytosol or entire organelles become sequestered in double membrane vesicles known as autophagosomes. ${ }^{40}$ Autophagosomes are subsequently fused with lysosomes, thereby generating single-membrane autophagolysosomes and leading to cargo degradation. Autophagy is an important catabolic mechanism that recycles building blocks for basal macromolecular synthesis when cells are under nutrient starvation conditions, degrades damaged organelles, and eliminates pathogens that invade cells. ${ }^{41}$ The extremity of this catabolic process leads to type II programmed cell death, also called autophagic cell death. ${ }^{39}$

Two recent studies implicated autophagy in driving T-cell death. One study found that HIV-1 envelope glycoproteins (Env), expressed at the surface of infected cells, induce death of uninfected CD4 ${ }^{+} \mathrm{T}$ cells by inducing autophagy and an accumulation of Beclin 1 via CXCR4. Blockade of autophagy at different steps, either by drugs (3-methyladenine and bafilomycin A1) or by siRNAs specific for Beclin 1/Atg6 and Atg7 genes, totally inhibited cell death. Furthermore, CD4 ${ }^{+}$ $T$ cells still underwent Env-mediated cell death with autophagic features when apoptosis was inhibited. Another study 


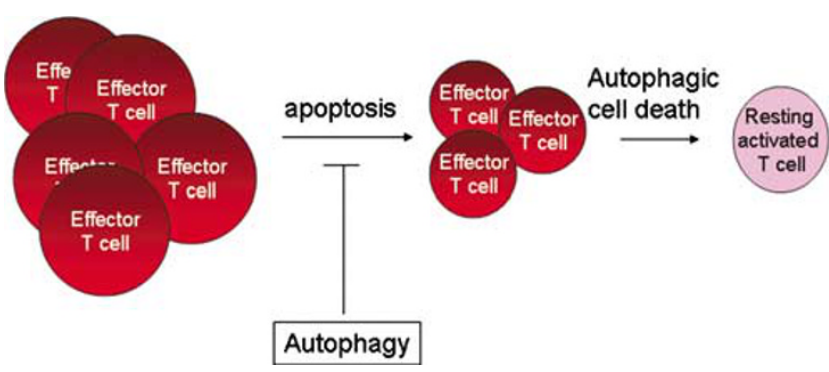

Figure 2 The dual roles of autophagy in T-cell life and death during growth factor withdrawal. Effector $T$ cells are susceptible to apoptosis when they are deprived of growth factors or nutrients. Autophagy plays a prosurvival role to antagonize apoptosis. However, autophagy induced in T cells that have survived apoptosis mediates T-cell death and leads to further reduction of T-cell number

demonstrated that cytokine withdrawal Th2 cell death is partially mediated by autophagic cell death. ${ }^{42}$ This finding is in line with another study involving immunization of mice with sheep red blood cells; there was a significant increase in both the number and the size of germinal centers in the spleens of beclin $1+/-$ as compared with beclin $1+/+$ mice. $^{43}$ Together, these studies suggest that autophagy inhibits adaptive humoral immune responses. Besides cell death, autophagy can be a prosurvival mechanism for T cells. In fact, peripheral $\mathrm{T}$ cells deficient of Atg5, a critical gene for autophagy, showed dramatic increase of spontaneous cell death. ${ }^{44}$ To reconcile the conclusions of these studies, we propose that autophagy plays dual roles in T-cell life and death. In the case of T-cell death during growth factor withdrawal, T cells first go through a quick death mediated by apoptosis. Autophagy plays a prosurvival role at this phase by degrading proteins to provide amino acids for the survival of $T$ cells. In T cells that have survived apoptosis, autophagy plays a pro-death role and causes further reduction of cell numbers (Figure 2).

\section{T-cell Death and Tolerance Induction by Tumor Antigens}

Adaptive immune response against tumors is initiated in the tumor draining lymph nodes (TDLNs) into which soluble tumor antigens, tumor fragments or apoptotic tumor cells drain or are carried by dendritic cells (DCs) infiltrating the tumor site. Many of these molecules are capable of providing the 'danger' signal required for $\mathrm{DC}$ stimulation and efficient presentation of tumor antigens to naïve T cells. ${ }^{45}$ If appropriately stimulated, naïve T cells become activated effector cells that can then migrate to the tumor site to kill cancer cells. However, it is now apparent that at least in advanced cancer, in TDLNs, DCs are prevented from proper maturation and activation ${ }^{46}$ or are actively immunosuppressive. These DCs, instead of activating T cells induce tolerance to tumor antigens (Figure 3). T-cell death is actively involved in the induction of tolerance in TDLNs.

Cross-tolerance to tumor antigens. Many tumor antigens are capable of eliciting T-cell responses when their expression levels get elevated above the normal threshold of detection, such as for example as a result of increased apoptosis of tumor cells. Tumor draining lymph nodes are

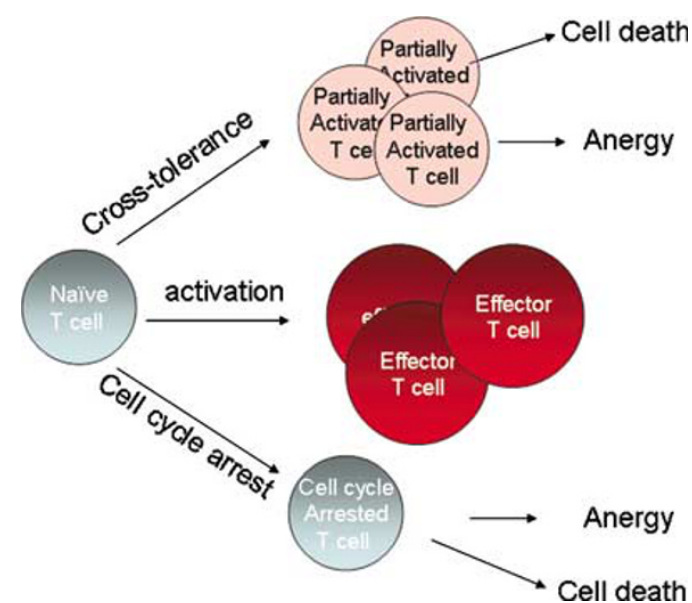

Figure 3 T cell fates after reacting with antigen presenting cells loaded with tumor antigens. After T cells interact with mature DCs, they differentiate into effector cells. When T cells encounter tolerogenic DC antigen-presenting cells, they become cell cycle arrested, and then either enter a nonresponsive state (anergy), or go through apoptosis. When T cells encounter immature DCs, they proliferate briefly, and then die or become anergic

believed to be the primary site where adaptive immune responses are initiated against tumor antigens. Tumorspecific naïve $\mathrm{CD}^{+}{ }^{+} \mathrm{T}$ cells can be stimulated by $\mathrm{DCs}$ ' presenting tumor antigens through $\mathrm{MHC}$ class II. Naïve $\mathrm{CD}^{+}{ }^{+} \mathrm{T}$ cells can also be stimulated by DCs' crosspresenting tumor antigens by MHC class $1 .{ }^{47}$ In many cases, in the tumor microenvironment, the presentation of tumor antigens is carried out by not fully activated, immature or immunosuppressed DCs leading to anergy or deletion of tumor-specific T cells. A similar problem can be encountered with vaccines, where tumor-induced immunosuppression of either DC or T-cell function leads to impaired responsiveness to vaccination. This type of tumor microenvironment triggered tolerance is called cross-tolerance. During crosstolerance, tumor antigens are presented by DCs and recognized by $\mathrm{T}$ cells. However, T-cell activation is suboptimal and their proliferation is not sustainable. Most tumor antigen-specific $T$ cells under these circumstances quickly disappear due to apoptosis. ${ }^{48,49}$ Therefore, programmed cell death is one pathway tumor cells exploit to deplete $T$ cells and to avoid immunosurveillance. ${ }^{48,49}$

Recent studies on the tolerance to tissue-associated selfantigens have shed light on the mechanism of cell death that is involved in cross-tolerance. Like tumor antigens, tissueassociated self-antigens are also cross-presented and the immune system utilizes this mechanism to achieve peripheral tolerance. Cross-presentation of self-antigen leads to deletion of naïve, autoreactive CD8 ${ }^{+}$T cells. $^{50}$ Both AICD and ACAD pathways were tested for their involvement in T-cell elimination in a model self-antigen expressed in the $\beta$ cells of the pancreas. ${ }^{51}$ Deletion of $\mathrm{CD}^{+} \mathrm{T}$ cells was prevented by overexpression of $\mathrm{Bcl}-2$, indicating that cross-tolerance was mediated by the Bcl-2 inhibitable pathway. In addition, Bim-deficient $T$ cells were not deleted in response to crosspresented self-antigen, strongly implicating $\mathrm{Bim}$ as the proapoptotic mediator of cross-tolerance. In contrast, the Fas pathway is not required for cross-tolerance. ${ }^{51}$ Based 
on the similarity between tissue-associated self-antigens and tumor antigens, it is reasonable to expect that $T$ cells specific for tumor antigens are tolerized by a similar mechanism. Therefore, ACAD is likely a main mechanism that leads to T-cell deletion during cross-tolerance. This is likely due to the lack of appropriate signals from APC after antigen recognition. These signals could be co-stimulatory signals or cytokines.

T-cell suppression by catabolizing tryptophan and arginine. Enzymes that regulate the availability of amino acids are involved in the induction of tolerance of tumor antigens. The enzyme indoleamine 2,3-dioxygenase (IDO), which catalyzes the rate-limiting step of tryptophan degradation along the kynurenine pathway, ${ }^{52}$ was shown to be induced in tolerogenic DCs such as plasmacytoid DCs ${ }^{52}$ and a subset of $\mathrm{CD} 8 \alpha^{+}$splenic DCs (Figure 3). ${ }^{53,54}$ An increased number of IDO-expressing cells in TDLNs correlates with a worse clinical outcome. ${ }^{52}$ The activity of IDO is critical for the tolerogenic functions of IDO ${ }^{+} \mathrm{DCs}^{52,54}$ IDO can be induced on DCs and macrophages either by reverse signaling of $\mathrm{B} 7-1$ and $\mathrm{B} 7-2$ or by Th1 cytokine IFN $\gamma .{ }^{54-56}$ Therefore, IDO is a self-limiting mechanism induced by Th1 responses.

Many human tumors also express IDO (Figure 4). Expression of IDO by immunogenic mouse tumor cells prevents their rejection by mice, which have been vaccinated against the same tumors. Injecting mice with an inhibitor of IDO can partly reverse such effect. ${ }^{57}$ As discussed earlier, IDO expression by tumor cells can also lead to T-cell death.

Both the reduction of local tryptophan levels and the production of tryptophan metabolites mediate the immunosuppressive effects of IDO. ${ }^{52}$ Naïve murine T cells or human total $T$ cells activated in chemically defined tryptophan-free media fail to synthesize DNA (Figure 3). ${ }^{58,59}$ Tryptophan deprivation caused cell cycle arrest of activated $T$ cells in mid-G1 phase. ${ }^{58,59}$ These arrested but activated naïve T cells cultured in tryptophan-free media are much more sensitive to Fas-mediated cell death ${ }^{58}$ when these cells are incubated with an agonistic anti-Fas antibody. Whether the Fas pathway is involved in deleting IDO-tolerized T cell in vivo remains to be carefully examined.

Tryptophan metabolites exert immunosuppressive effects on T cells by inhibiting proliferation or by causing cell death. ${ }^{60}$ 3-Hydroxyantranylic acid (3-HAA) and quinolinic acid (QUIN) are L-tryptophan metabolites formed along the metabolic pathway known as the kynurenine pathway, in which IDO catalyzes the initial and rate-limiting step. ${ }^{61}$ 3-HAA and QUIN induced the apoptosis in vitro of murine thymocytes and of Th1 but not Th2 cells. Fas is dispensable for the cytotoxic effects. ${ }^{62}$ The pan-caspase inhibitor zVAD and the caspase 8 inhibitor were able to block cell death triggered by 3-HAA and QUIN. Cytochrome $c$ release was observed in T cell treated with 3-HAA and QUIN, suggesting the involvement of the mitochondrial death pathway. ${ }^{62}$ Therefore, one of the mechanisms of IDO-mediated suppression is due to the killing of Th1 cells by tryptophan metabolites.

L-arginine plays an important role in regulating immune responses by myeloid suppressor cells. ${ }^{63}$ It is metabolized in macrophages, endothelial cells, hepatocytes, kidney cells, and certain tumors by three enzymatic pathways, the inducible nitric oxide synthase (iNOS), arginase I (ASE I), and arginase II (ASE II). ${ }^{63}$ L-arginine is metabolized by iNOS to produce citrulline and nitric oxide, which is one of the major cytotoxic mechanisms in these cells. ${ }^{64}$ Alternatively, ASE I and ASE II metabolize L-arginine to L-ornithine and urea. ${ }^{65}$ The expression of ASE I and iNOS in murine myeloid suppressor cells is differentially regulated by Th1 and Th2 cytokines. $^{66,67}$ IFN- $\gamma$ upregulates iNOS, whereas IL-4, IL-10, and IL-13 induce ASE I. ${ }^{66,67}$ Many tumors can induce myeloid suppressor cells within themselves and in the peripheral immune system. Myeloid suppressor cells are believed to inhibit tumor-specific immune responses by depleting arginine and generating NO. Reduced levels of arginine inhibit antigenspecific proliferation of $\mathrm{T}$ cells. In addition, both iNOS and ASEI are important in causing T-cell death. However, the detailed mechanism of cell death is not known. In light of studies on IDO, the arrest of cell cycle can render T cells more susceptible to cell death stimuli such as FasL.

The availability of amino acids is well known to regulate autophagy. ${ }^{68}$ High levels of tryptophan reduce autophagy and low levels likely will induce autophagy in T cells. Arginine does not regulate autophagy, at least in liver cells. ${ }^{68}$ However, its role in T-cell autophagy should be examined because $\mathrm{T}$ cells seem to be quite sensitive to levels of arginine. Interestingly, the lack of either arginine or tryptophan induces the activity of Gcn2 which mediates the suppressive effect on T cells. ${ }^{52,69}$ Another member of the Gcn2 family PKR was shown in macrophages to be critical in inducing autophagy. ${ }^{70}$ Therefore, Gcn2 is expected to play a similar role in $\mathrm{T}$ cells. Autophagy is a stress response to the lack of nutrients such as amino acids. ${ }^{68}$ Autophagy is likely important for the short-term survival of $T$ cells in TDLNs and the tumor microenvironment because this process generates essential amino acids by degrading cellular protein for essential functions of cells. However, extensive and sustained autophagy will likely lead to eventual cell death. ${ }^{39}$ Therefore, it is also probable that autophagy will be involved in mediating cell death when T cells are incubated without these essential amino acids for prolonged periods of time.

CD4 $^{+} \mathbf{T}$ cells help CTL responses. Because killer $\mathrm{T}$ cells can directly destroy cancer cells, boosting antitumor CTL responses is the priority for immunotherapy of cancer. Help provided by $\mathrm{CD} 4{ }^{+} \mathrm{T}$ cells is considered to 'license' DCs to activate CD8 ${ }^{+}$T cells. $^{71}$ Therefore, an effective CD4 ${ }^{+} \mathrm{T}$-cell response is believed to be essential to prevent tolerance induction by tumor antigens. ${ }^{72}$ Recent studies revealed that during acute viral infections, CD4 help has a dramatic impact on the memory recall responses of $\mathrm{CD}^{+}{ }^{+} \mathrm{T}_{\text {cells. }}{ }^{71} \mathrm{CD} 4^{+}$ $\mathrm{T}$ cells are not required for primary expansion of $\mathrm{CD}^{+} \mathrm{T}$ cells and their differentiation into cytotoxic effectors in response to acute infection. However, secondary CTL expansion, a hallmark of adaptive immune response, is dependent on the presence of $\mathrm{CD} 4{ }^{+} \mathrm{T}$ cells during, but not after, priming. ${ }^{71}$ The genetic program imprinted on $\mathrm{CD} 8^{+} \mathrm{T}$ cells by helper $T$ cells appears to involve apoptotic pathways. Addition of caspase inhibitors quinoline-Val-Asp-CH2difluorophenoxy (qVD-OPh) and benzyloxycarbonyl-Val-AlaAsp-fluoromethyl ketone (zVAD-fmk) restores secondary expansion of $\mathrm{CD}^{+} \mathrm{T}$ cells primed in the absence of CD4 
help. Neither expression of Bcl-2 or Bcl-xL nor the absence of Fas (Ipr/lpr) or TNFR (TNFR1-/-) helped recover secondary expansion in helpless $\mathrm{CD}^{+}{ }^{+} \mathrm{T}$ cells. Effector $\mathrm{T}$ cells from Trail-deficient mice, however, proliferated in response to secondary stimulation regardless of $\mathrm{CD}^{+}{ }^{+}$help. Therefore, $\mathrm{CD}^{+}{ }^{+} \mathrm{T}$ cells help program $\mathrm{CD}^{+} \mathrm{T}$ cells to suppress TRAIL-mediated AICD during memory responses. Lack of $\mathrm{CD}^{+}{ }^{+} \mathrm{T}$ cells help is also involved in the deletion of CTLs in the chronic infection setting. ${ }^{71}$ The persistent presence of low levels of antigens in these settings is similar to some cancers. Therefore, it is possible that CTLs against tumor antigens are deleted when helper responses are absent. ${ }^{73}$ Therefore, tolerance of $\mathrm{CD}^{+}{ }^{+} \mathrm{T}$ cells by tumor will affect $\mathrm{CD}^{+} \mathrm{T}$-cell recall responses by the death receptor pathway.

\section{Killing Effector $\mathrm{T}$ cells by Tumors}

Tumor produced effector molecules that kill tumorinfiltrating $\mathbf{T}$ cells. Cancer-specific effector $\mathrm{T}$ cells migrate to and infiltrate tumors. Killer T cells can recognize and kill their target transformed cells. Th1 cells can either serve as killer cells or help develop a delayed type hypersensitivity reaction within a tumor. The presence of Th1-type cells marker has been associated with positive prognosis in many type of cancers. Therefore, the presence of functional effector $T$ cells within tumor mass is important for successful immunosurveillance. FasL expressed on the surface of $\mathrm{T}$ cells has an important role in mediating the death of Fas-expressing tumor cells. However, many cancer cells have evolved to develop resistance to FasL/Fasmediated killing. Moreover, they gained the ability to use FasL/Fas pathway to counteract T cells (Figure 4).

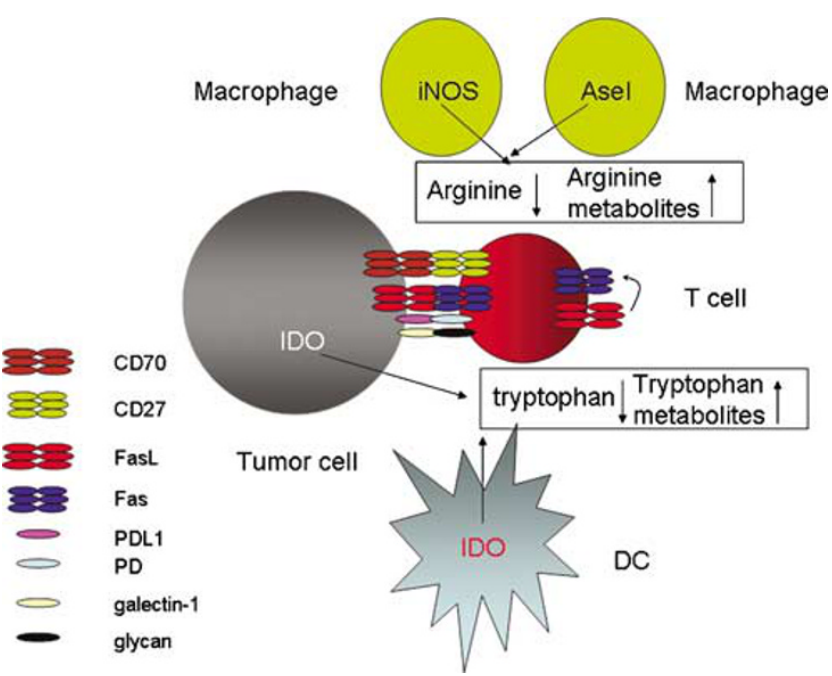

Figure 4 T-cell death in the tumor microenvironment. Tumors contribute to Tcell death by expressing death receptors, PDL1, galectin-1, etc. In addition, cancer cells and DCs express IDO that depletes tryptophan and generate tryptophan metabolites to cause T-cell death. Moreover, tumor-infiltrating macrophages upregulate iNOS and Asel, which deplete arginine and generate arginine metabolites to cause T-cell death. Depletion of amino acids might also lead to autophagy-mediated cell death
Many studies have demonstrated FasL expression in many diverse types of human cancer, where it may contribute to tumor immune evasion. For example, FasL expression in colon cancer has been demonstrated by several groups. ${ }^{74}$ FasL expression was also found in head and neck cancer, ovarian cancer, melanoma, and renal cell carcinoma. ${ }^{74}$ The expression of FasL on tumors coupled with the fact that many tumors are resistant to Fas-mediated apoptosis support the hypothesis that tumors use FasL to launch a preemptive attack against activated T cells as a way to avoid destruction. ${ }^{75}$ Therefore, FasL helps make tumor tissues an immunoprivileged site.

There is a wealth of experimental evidence that FasL on tumors is functional. FasL expression has been found on tumor cells in areas of intense lymphocytic infiltrates. Many apoptotic lymphocytes were observed in these regions. This same phenomenon has been found in many cancers such as esophageal tumors, head and neck cancer, and ovarian carcinoma. ${ }^{74}$ In vitro, co-culture of FasL ${ }^{+}$tumor cells with $\mathrm{T}$ lymphocytes induced apoptosis in activated Fas + lymphocytes. ${ }^{76,77}$ Apoptosis was blocked by anti-FasL Abs or Fas-Fc protein capable of binding and neutralizing FasL. ${ }^{76}$

FasL expressed on tumors can also be secreted. Because the soluble form is not a potent inducer of apoptosis, conversion from the membrane bound form to the soluble form was believed to downregulate the FasL function. ${ }^{74}$ In contrast, injection of soluble FasL into mice caused liver damage indicating functional potential. ${ }^{78}$ This discrepancy was resolved by a study that found that extracellular matrix proteins interact with soluble CD95L and potentiated its proapoptotic activity. ${ }^{79}$ Therefore, the soluble form of FasL made by tumor cells can further promote the suppressive tumor microenvironment by binding to the extracellular matrix. This FasL-decorated extracellular matrix would be fully capable of mediating the killing of tumor-specific effector $\mathrm{T}$ cells infiltrating the tumor site.

It has already been established that FasL-triggered T-cell death only happens in highly activated effector $T$ cells upon encountering their antigens. ${ }^{80,81}$ Resting $T$ cells are not susceptible to FasL-driven apoptosis. ${ }^{80,81}$ Therefore, expressing FasL on the surface of tumor cells or on the extracellular matrix surrounding the tumor will ensure the most efficient elimination of tumor-specific highly active effector $T$ cells, but preserve less active or suppressed T cells in the tumor mass. Therefore, by selection of perhaps initially only a small number of tumor cells with FasL expression, tumors can grow progressively by weakening the immune responses to the point of immune evasion.

The significance of tumor-FasL counter attack is supported by the clear prognostic value that FasL expression has in many cancers. Analysis of the expression of FasL mRNA and protein in paired tissue samples of normal colonic mucosa $(\mathrm{N})$, primary colorectal carcinomas $(T)$, and their metastases (M) revealed that metastasizing subpopulations of colorectal tumor cells express FasL more frequently than the primary carcinomas. ${ }^{74}$ The status of Fas and Fas ligand expression had prognostic significance for disease-free survival and recurrence of hepatocellular carcinoma. ${ }^{74}$ In colorectal carcinoma, the cases with a high apoptosis index of tumor infiltration $\mathrm{T}$ cells had a significantly poorer prognosis than 
those with a low apoptosis index. ${ }^{74}$ Similar findings have been reported in breast cancers. ${ }^{74}$

The FasL serving as immune evasion mechanism for tumors is consistent with its role in maintenance of the immune-privileged status in the eye ${ }^{82}$ and testis. ${ }^{83}$ To confound the situation, several studies suggested that FasL on tumors could be proinflammatory. Transplanted tumor cells that express FasL were quickly rejected ${ }^{84,85}$ in mice compared to tumors lacking this molecule. It turned out that the rejection in these cases was largely mediated by infiltrating neutrophils. ${ }^{84}$ These results seem to be contradictory to the hypothesis that FasL is critical for tumor immune evasion. However, it is now clear that different tumors create different microenvironments characterized by the presence or absence of various cytokines. One of the important cytokines that may determine the ultimate importance of the FasL expression is TGF $\beta 1$. One study showed that tumor rejection by neutrophils was prevented by adding TGF $\beta 1$ to the tumor microenvironment. ${ }^{86}$ Therefore, FasL and TGF $\beta$ together generate a different microenvironment than either one alone or in combination with other cytokines. In most cases, however, the cytokine combinations promote immunologic tolerance.

Signals other than Fas also mediate T-cell killing by malignant cells (Figure 4). The TNF receptor family member CD27 is constitutively expressed on the majority of T cells and on subsets of antigen-experienced B cells, NK cells, and hematopoietic progenitor cells. ${ }^{87}$ In contrast, expression of its ligand CD70 is tightly regulated and only transiently expressed on activated T cells and DCs. ${ }^{87}$ Diminished expansion of antigen-specific $\mathrm{T}$ cells in primary and memory $\mathrm{T}$-cell responses to influenza virus infection was observed in CD27-/- mice. ${ }^{87}$ However, chronic activation of CD27 in mice resulted in depletion of the naïve T-cell compartment and subsequent death from opportunistic infection. ${ }^{88}$ These data again demonstrate that chronic overstimulation of an otherwise activating signaling pathway tolerizes immune responses. AICD mediated by CD27 signaling is believed to cause T-cell deletion. Siva, a proapoptotic protein, can bind to the cytoplasmic tail of CD27 and has been implicated in apoptosis induction through CD27 via a caspase-dependent mitochondrial pathway. ${ }^{89}$ Immune stimulatory signals transduced via CD27 are mediated by the adaptor proteins TNF receptor-associated factor 2 and $5 .^{87}$ CD70 protein was detected by immunocytochemistry in glioblastomas and anaplastic astrocytomas. CD27 expression was not detected in any glioma cell line. ${ }^{90}$ Glioblastoma cell lines cocultured with $\mathrm{T}$ cells induced lymphocyte death. CD70 is responsible for initiating T-cell death via the receptor-dependent pathway. ${ }^{90}$ Therefore, at least some cancer cells mediate immunosuppression by promoting T-cell death via tumorassociated CD70.

PDL1 is also highly expressed in various tumor tissues. Blocking PDL1 function has been shown to relieve immune exhaustion in chronic infection. Tumors can be quickly eliminated when PDL1 function is neutralized. ${ }^{91}$ Patients with PDL1-positive tumors had poor prognosis and shorter survival. ${ }^{92}$ PDL1 induction of T-cell death was one of several proposed mechanisms for its role in immune suppression. ${ }^{91}$ This is supported by the inverse correlation between PDL1 expression on tumor cells and the number of tumor-infiltrating lymphocytes (TILs). ${ }^{93}$

Besides antigen-specific and co-stimulatory receptors, T-cell surface glycans can react with tumor or tumor stromaassociated lectins to cause T-cell apoptosis. One such lectin, galectin-1, has a relatively low affinity for single $N$-acetyllactosamine sequences; however, galectin-1 binds with high avidity to glycans containing multiple $N$-acetyllactosamine units and preferentially binds glycoproteins containing linear polylactosamine sequences. ${ }^{94}$ Galectin-1 sensitizes human T cells to Fas (CD95)/caspase 8-mediated cell death. ${ }^{95}$ Human squamous laryngeal cancer patients that display high levels of galectin-1 have worse prognoses than those with laryngeal cancers with low levels of galectin-1 expression. ${ }^{96}$ Elevated expression of galectin-1 in carcinoma-associated stroma predicts poor outcome in prostate carcinoma patients. ${ }^{97} \mathrm{Head}$ and neck squamous cell carcinomas that stained strongly for galectin-1 had significantly lower levels of $T$ cells (as assessed by CD3 staining) than those with negative or weak staining for galectin-1. Galectin-1 expression negatively correlated with survival in head and neck cancer patients. ${ }^{98}$ All these results are consistent with the idea that galectin-1 contributes to tumor immune evasion by killing effector T cells.

AICD and T-cell suicide in the tumor microenvironment. Effector T-cell suicide is an important self-limiting mechanism. AICD is a major mechanism that eliminates hyperactivated Th1 cells and $\mathrm{CD}^{+}{ }^{+} \mathrm{T}$ cells from the sites of an immune response, thereby limiting immune pathology. Persistent triggering of TCR is believed to upregulate FasL, caspases and downregulate $\mathrm{Bcl}-2$ and FLIP, the inhibitor of apoptosis, which then leads to massive T-cell apoptosis. $^{81}$ The tumor mass is not only an immune suppressive environment, but is also a source of overwhelming amounts of antigens that chronically stimulate $\mathrm{T}$ cells that infiltrate it. Chronic activation is believed to result in exhaustion of $T$ cells such as in the case of HIV infection and HBV infection. One mechanism of exhaustion is T-cell depletion, very likely through AICD. Ample evidence suggests that $\mathrm{T}$-cell suicide is an important mechanism whereby highly cytotoxic $T$ cells are eliminated within tumors. In a melanoma study, it was found that melanoma tumor cells caused the apoptotic death of tumorspecific T cells only upon specific MHC class I-restricted recognition, suggesting cell death is initiated by recognition of tumor antigens. This death was entirely blockable by the addition of a neutralizing antibody against the Fas death receptor (APO-1, CD95). Contrary to the view that tumor cells cause the death of antitumor $T$ cells by expressing Fas ligand (FasL), data in this study suggested that FasL was expressed by $\mathrm{T}$ lymphocytes upon activation, not on melanoma. ${ }^{99}$ Another study showed that Fas or FasLdeficient tumor-specific Th1 cells survive better in tumorbearing mice. Moreover, Fas- or FasL-deficient tumorspecific Th1 cells were more effective than wild-type Th1 cells at eliminating tumors. ${ }^{100}$ These results imply that Fasmediated activation-induced cell death could be a limiting factor in effective $\mathrm{T}$ cell-mediated immunosurveillance or immunotherapy. 


\section{Contraction of Effector T cells and Maintenance of Memory T cells}

The acute immune responses such as those induced by cancer vaccines or acute infection can be divided into four relatively distinct phases. The first, activation phase is critically dependent on productive engagement between naïve $\mathrm{T}$ cells and mature DCs. ${ }^{37}$ The second phase, expansion, usually lasts 5-8 days in mouse acute infectious models, during which Ag-specific T-cell numbers increase dramatically. ${ }^{37}$ Clonal expansion is also associated with differentiation to effector $\mathrm{T}$ cells that migrate into various tissues. ${ }^{37}$ Once pathogens are eliminated, the third phase, contraction, follows suite and $90-95 \%$ of effector cells are eliminated over the ensuing weeks. ${ }^{37}$ The final phase is the generation and maintenance of the long-lived memory T-cell pool. $^{37}$ It is believed that contraction and homeostatic maintenance of both memory $\mathrm{CD}^{+}{ }^{+} \mathrm{T}$ cells and $\mathrm{CD} 8^{+} \mathrm{T}$ cells are largely dependent on IL-15 in normal animals and also on IL-7 in lymphopenic animals. ${ }^{101}$ The turn-over of memory T cells is slow and it is believed that the main function of IL-7 and IL-15 is to maintain the survival of memory T cells. ACAD, not AICD, is antagonized by IL-15 and IL-7. Consistent with this idea, bim-/- mice had a delayed contraction phase after SEB challenge. ${ }^{34}$ In contrast, the death receptor pathway is not required for diminishing $T$-cell number in the same experimental setting. In addition, the HSV-specific $\mathrm{CD}^{+}{ }^{+} \mathrm{T}$ cells persisted in bim/ mice long after HSV clearance. ${ }^{35}$ Collectively, these studies suggested that the Bcl-2 pathway is important for memory generation and maintenance by cytokines. The remaining question is whether these bim/ 'memory' $T$ cells are true memory $T$ cells or effector $T$ cells rescued through lack of apoptosis.

Another cytokine that regulates contraction of effector $T$ cells is IFN $\gamma$, the hallmark cytokine made by Th1 cells and $\mathrm{CD}^{+}{ }^{+} \mathrm{T}$ cells. IFN $\gamma$-deficient mice generated more similar numbers of antigen-specific Th1 cells and $\mathrm{CD}^{+} \mathrm{T}$ cells during infection, DC vaccination, autoimmune EAE, and tumor vaccination. ${ }^{102-104}$ However, there were significant delays of contraction of IFN $\gamma$-deficient T cells. ${ }^{102-104}$ The memory responses against tumor vaccination were much more potent when IFN $\gamma$ signaling was blocked. ${ }^{104}$ Two possible mechanisms were proposed. IFN $\gamma$ can induce caspase expression in $\mathrm{T}$ cells. Overexpression of caspase 8 in IFN $\gamma$-deficient Th1 cells recovered their sensitivity to AICD. However, since AICD is not involved in contraction, the more likely explanation is that caspase 3, which is also upregulated by IFN $\gamma$, is able to mediate the $\mathrm{Bcl}-2$ pathway of death. The other mechanism is that higher portions of IFN $\gamma$-deficient $\mathrm{CD}^{+}{ }^{+} \mathrm{T}$ cells expressed high levels of IL-7r. ${ }^{105}$ These IL-7r-positive effector T cells were shown to be the precursors of memory cells. ${ }^{106}$

\section{Therapeutic Implications}

Evidence accumulated over many years of studying tumorimmune system interactions in animal models and in cancer patients has singled out the adaptive immunity, in particular tumor-specific T cells as an important mechanism of tumor surveillance as well as tumor rejection. It is thus not surprising that tumors have hijacked many normal mechanisms that maintain health by eliminating effector $T$ cells and maintaining immune tolerance, and use them to achieve immune evasion and uncontrolled growth. The importance of characterizing specific mechanisms that different patients or different tumors employ is related to the need to improve the efficacy of tumor immunotherapy. ${ }^{107}$ In the case of cancer vaccines, the goal is to boost already existing tumorspecific T-cell responses or attempt to prime additional responses. Clearly, the ability to do that is predicated on several conditions: (1) antigen-presenting cells need to be responsive to maturation and activation signals in order to effectively cross-present antigens from the vaccine to $T$ cells; (2) tumor-antigen-specific memory T cells need to be capable of expansion upon antigen recognition; (3) tumor-antigenspecific effector $T$ cells need to be able to migrate to tumor sites and survive long enough to make beneficial cytokines or kill tumor cells. Every one of these, if not all, is interfered by the tumor or its products, as we discussed above. One way of dealing with the competence of APCs has been to take, expand them in vitro, properly mature and activate them, load them with antigen and inject back into the patient. ${ }^{108}$ Even if this labor intensive, customized and personalized approach to vaccination would become broadly feasible, the problem of $\mathrm{T}$-cell death in the tumor microenvironment will remain. The success of inducing good effector $\mathrm{T}$ cells at the vaccination site will be compromised by the effect of various immunoinhibitory forces in the tumor microenvironment, such as FasL, IDO, TGF- $\beta$, and others. Adoptive transfer of large numbers of tumor-specific effector $T$ cells, recently generating some enthusiasm due to evidence of therapeutic efficacy, is equally problematic in the context of tumor-induced immunosuppression. There is no experimental evidence to show that these cells are less susceptible to the effects of various death signals present in the tumor, than $\mathrm{T}$ cells elicited by vaccination. The only advantage might be the much larger cell number that can be transferred compared to the numbers that can be elicited through vaccination, thus assuring some therapeutic effect before all the cells disappear. The latest report shows that adoptively transferred $\mathrm{T}$ cells persist in the patients long term, however this does not correlate with the therapeutic effect. $^{109}$

One important factor that we have not discussed and for which there is not sufficient information is the importance of disease stage and immunosuppression. Most of the T-cell killing mechanisms described above were initially found in late stage tumors. By definition, however, even tumors designated by pathologists to be early stage may be late stage when considered by the immune system. Certainly, even earlystage tumors have evaded immune control, suggesting that they have acquired many immunosuppressive characteristics. If experiments in the future show that $\mathrm{T}$ cells are much more effective against early disease and that immunosuppressive factors are fewer, it would stand to reason that both active and passive T-cell therapy for late-stage disease should be strongly discouraged and efforts should be expanded to move such therapies to early disease. If, however, both early- and late-stage cancers have the same immunosuppressive potential, efforts are best spent in developing these therapies for the setting of premalignant 
lesions to stop their progression to full blown immunosuppressive tumors that kill T cells. ${ }^{19,110}$

1. Ehrlich P. Ueber den jetzigen stand der Karzinomforschung. Ned Tijdschr Geneeskd 1909; 5: 273-290.

2. Old LJ, Boyse EA. Immunology of experimental tumors. Annu Rev Med 1964; 15: 167-186.

3. Burnet FM. The concept of immunological surveillance. Prog Exp Tumor Res 1970; 13: $1-27$.

4. Burnet M. Immunological factors in the process of carcinogenesis. Br Med Bull 1964; 20: 154-158.

5. Graziano DF, Finn OJ. Tumor antigens and tumor antigen discovery. Cancer Treat Res 2005; 123: 89-111.

6. Dighe AS, Richards E, Old LJ, Schreiber RD. Enhanced in vivo growth and resistance to rejection of tumor cells expressing dominant negative IFN gamma receptors. Immunity 1994; 1: 447-456.

7. Kaplan DH, Shankaran V, Dighe AS, Stockert E, Aguet M, Old LJ et al. Demonstration of an interferon gamma-dependent tumor surveillance system in immunocompetent mice. Proc Natl Acad Sci USA 1998; 95: 7556-7561.

8. Street SE, Trapani JA, MacGregor D, Smyth MJ. Suppression of lymphoma and epithelial malignancies effected by interferon gamma. J Exp Med 2002; 196: 129-134.

9. Gao Y, Yang W, Pan M, Scully E, Girardi M, Augenlicht LH et al. Gamma delta T cells provide an early source of interferon gamma in tumor immunity. J Exp Med 2003; 198: 433-442.

10. Girardi M, Oppenheim DE, Steele CR, Lewis JM, Glusac E, Filler R et al. Regulation of cutaneous malignancy by gammadelta T cells. Science 2001; 294: 605-609.

11. Shankaran V, Ikeda H, Bruce AT, White JM, Swanson PE, Old LJ et al. IFNgamma and lymphocytes prevent primary tumour development and shape tumour immunogenicity. Nature 2001; 410: 1107-1111.

12. Pages F, Berger A, Camus M, Sanchez-Cabo F, Costes A, Molidor R et al. Effector memory T cells, early metastasis, and survival in colorectal cancer. N Engl J Med 2005; 353: 2654-2666.

13. Galon J, Costes A, Sanchez-Cabo F, Kirilovsky A, Mlecnik B, Lagorce-Pages C et al. Type, density, and location of immune cells within human colorectal tumors predict clinical outcome. Science 2006; 313: 1960-1964.

14. Hanahan D, Weinberg RA. The hallmarks of cancer. Cell 2000; 100: 57-70.

15. Zitvogel L, Tesniere A, Kroemer G. Cancer despite immunosurveillance: immunoselection and immunosubversion. Nat Rev Immunol 2006; 6: 715-727.

16. Willimsky $G$, Blankenstein T. Sporadic immunogenic tumours avoid destruction by inducing T-cell tolerance. Nature 2005; 437: 141-146.

17. Dunn GP, Bruce AT, Ikeda H, Old LJ, Schreiber RD. Cancer immunoediting: from immunosurveillance to tumor escape. Nat Immunol 2002; 3: 991-998.

18. Zou W. Immunosuppressive networks in the tumour environment and their therapeutic relevance. Nat Rev Cancer 2005; 5: 263-274.

19. Finn OJ. Cancer vaccines: between the idea and the reality. Nat Rev Immunol 2003; 3 : 630-641.

20. Marrack P, Kappler J. Control of T cell viability. Annu Rev Immunol 2004; 22: 765-787.

21. Kerr JF, Wyllie AH, Currie AR. Apoptosis: a basic biological phenomenon with wideranging implications in tissue kinetics. Br J Cancer 1972; 26: 239-257.

22. Fadok VA, Savill JS, Haslett C, Bratton DL, Doherty DE, Campbell PA et al. Different populations of macrophages use either the vitronectin receptor or the phosphatidylserine receptor to recognize and remove apoptotic cells. J Immunol 1992; 149: 4029-4035.

23. Shi Y. Mechanisms of caspase activation and inhibition during apoptosis. Mol Cell 2002; 9: $459-470$.

24. Hildeman DA, Zhu Y, Mitchell TC, Kappler J, Marrack P. Molecular mechanisms of activated T cell death in vivo. Curr Opin Immunol 2002; 14: 354-359.

25. Lenardo $M$, Chan KM, Hornung $F$, McFarland $H$, Siegel R, Wang $J$ et al. Mature $T$ lymphocyte apoptosis - immune regulation in a dynamic and unpredictable antigenic environment. Annu Rev Immunol 1999; 17: 221-253.

26. Krammer PH. CD95's deadly mission in the immune system. Nature 2000; 407: 789-795.

27. Lakhani SA, Masud A, Kuida K, Porter Jr GA, Booth CJ, Mehal WZ et al. Caspases 3 and 7: key mediators of mitochondrial events of apoptosis. Science 2006; 311: 847-851.

28. Siegel RM, Chan FK, Chun HJ, Lenardo MJ. The multifaceted role of Fas signaling in immune cell homeostasis and autoimmunity. Nat Immunol 2000; 1: 469-474.

29. Sabelko-Downes KA, Cross AH, Russell JH. Dual role for Fas ligand in the initiation of and recovery from experimental allergic encephalomyelitis. J Exp Med 1999; 189: 1195-1205.

30. Strasser A. The role of BH3-only proteins in the immune system. Nat Rev Immunol 2005; 5: 189-200.

31. Veis DJ, Sorenson CM, Shutter JR, Korsmeyer SJ. Bcl-2-deficient mice demonstrate fulminant lymphoid apoptosis, polycystic kidneys, and hypopigmented hair. Cell 1993; 75: 229-240.

32. Bouillet P, Metcalf D, Huang DC, Tarlinton DM, Kay TW, Kontgen F et al. Proapoptotic Bcl-2 relative Bim required for certain apoptotic responses, leukocyte homeostasis, and to preclude autoimmunity. Science 1999; 286: 1735-1738.

33. Strasser A, Pellegrini M. T-lymphocyte death during shutdown of an immune response. Trends Immunol 2004; 25: 610-615.
34. Hildeman DA, Zhu Y, Mitchell TC, Bouillet P, Strasser A, Kappler J et al. Activated T cell death in vivo mediated by proapoptotic bcl-2 family member bim. Immunity 2002; 16: 759-767.

35. Pellegrini M, Belz G, Bouillet $P$, Strasser A. Shutdown of an acute T cell immune response to viral infection is mediated by the proapoptotic Bcl-2 homology 3-only protein Bim. Proc Natl Acad Sci USA 2003; 100: 14175-14180.

36. Lu B, Zagouras P, Fischer JE, Lu J, Li B, Flavell RA. Kinetic analysis of genomewide gene expression reveals molecule circuitries that control $T$ cell activation and Th1/2 differentiation. Proc Natl Acad Sci USA 2004; 101: 3023-3028.

37. Seder RA, Ahmed R. Similarities and differences in CD4+ and CD8+ effector and memory T cell generation. Nat Immunol 2003; 4: 835-842.

38. Lu B. The molecular mechanisms that control function and death of effector CD4+ T cells. Immunol Res 2006; 36: 275-282.

39. Clarke PG. Developmental cell death: morphological diversity and multiple mechanisms. Anat Embryol (Berl) 1990; 181: 195-213.

40. Ohsumi Y. Molecular dissection of autophagy: two ubiquitin-like systems. Nat Rev Mol Cell Biol 2001; 2: 211-216.

41. Levine B, Deretic V. Unveiling the roles of autophagy in innate and adaptive immunity. Nat Rev Immunol 2007; 7: 767-777.

42. Li C, Capan E, Zhao Y, Zhao J, Stolz D, Watkins SC et al. Autophagy is induced in CD4+ $T$ cells and important for the growth factor-withdrawal cell death. J Immunol 2006; 177: 5163-5168.

43. Qu X, Yu J, Bhagat G, Furuya N, Hibshoosh $\mathrm{H}$, Troxel A et al. Promotion of tumorigenesis by heterozygous disruption of the beclin 1 autophagy gene. J Clin Invest 2003; 112: 1809-1820.

44. Pua HH, Dzhagalov I, Chuck M, Mizushima N, He YW. A critical role for the autophagy gene Atg5 in T cell survival and proliferation. J Exp Med 2007; 204: 25-31.

45. Rock KL, Shen L. Cross-presentation: underlying mechanisms and role in immune surveillance. Immunol Rev 2005; 207: 166-183.

46. Shurin MR, Shurin GV, Lokshin A, Yurkovetsky ZR, Gutkin DW, Chatta G et al. Intratumoral cytokines/chemokines/growth factors and tumor infiltrating dendritic cells: friends or enemies? Cancer Metast Rev 2006; 25: 333-356.

47. Bevan MJ. Cross-priming for a secondary cytotoxic response to minor $\mathrm{H}$ antigens with $\mathrm{H}-2$ congenic cells which do not cross-react in the cytotoxic assay. J Exp Med 1976; 143: 1283-1288.

48. Cuenca A, Cheng F, Wang H, Brayer J, Horna P, Gu L et al. Extra-lymphatic solid tumor growth is not immunologically ignored and results in early induction of antigen-specific Tcell anergy: dominant role of cross-tolerance to tumor antigens. Cancer Res 2003; 63 9007-9015

49. Drake CG, Doody AD, Mihalyo MA, Huang CT, Kelleher E, Ravi S et al. Androgen ablation mitigates tolerance to a prostate/prostate cancer-restricted antigen. Cancer Cell 2005; 7: 239-249.

50. Kurts C, Kosaka H, Carbone FR, Miller JF, Heath WR. Class I-restricted crosspresentation of exogenous self-antigens leads to deletion of autoreactive CD8(+) T cells. J Exp Med 1997; 186: 239-245.

51. Davey GM, Kurts C, Miller JF, Bouillet P, Strasser A, Brooks AG et al. Peripheral deletion of autoreactive CD8 $\mathrm{T}$ cells by cross presentation of self-antigen occurs by a Bcl-2inhibitable pathway mediated by Bim. J Exp Med 2002; 196: 947-955.

52. Munn DH, Mellor AL. Indoleamine 2,3-dioxygenase and tumor-induced tolerance. J Clin Invest 2007; 117: 1147-1154.

53. Grohmann U, Fallarino F, Bianchi R, Belladonna ML, Vacca C, Orabona C et al. IL-6 inhibits the tolerogenic function of $\mathrm{CD} 8$ alpha+ dendritic cells expressing indoleamine 2,3 dioxygenase. J Immunol 2001; 167: 708-714.

54. Orabona C, Puccetti P, Vacca C, Bicciato S, Luchini A, Fallarino F et al. Toward the identification of a tolerogenic signature in IDO-competent dendritic cells. Blood 2006; 107 2846-2854.

55. Koide $\mathrm{Y}$, Yoshida $\mathrm{A}$. The signal transduction mechanism responsible for gamma interferon-induced indoleamine 2,3-dioxygenase gene expression. Infect Immun 1994; 62: 948-955.

56. Carlin JM, Borden EC, Sondel PM, Byrne Gl. Interferon-induced indoleamine 2,3dioxygenase activity in human mononuclear phagocytes. J Leukoc Biol 1989; 45: 29-34.

57. Uyttenhove C, Pilotte L, Theate I, Stroobant V, Colau D, Parmentier N et al. Evidence for a tumoral immune resistance mechanism based on tryptophan degradation by indoleamine 2,3-dioxygenase. Nat Med 2003; 9: 1269-1274.

58. Lee GK, Park HJ, Macleod M, Chandler P, Munn DH, Mellor AL. Tryptophan deprivation sensitizes activated T cells to apoptosis prior to cell division. Immunology 2002; 107 $452-460$.

59. Munn DH, Shafizadeh E, Attwood JT, Bondarev I, Pashine A, Mellor AL. Inhibition of T cell proliferation by macrophage tryptophan catabolism. J Exp Med 1999; 189: 1363-1372.

60. Terness P, Bauer TM, Rose L, Dufter C, Watzlik A, Simon $\mathrm{H}$ et al. Inhibition of allogeneic $T$ cell proliferation by indoleamine 2,3-dioxygenase-expressing dendritic cells: mediation of suppression by tryptophan metabolites. J Exp Med 2002; 196: 447-457.

61. Taylor MW, Feng GS. Relationship between interferon-gamma, indoleamine 2,3dioxygenase, and tryptophan catabolism. FASEB J 1991; 5: 2516-2522.

62. Fallarino F, Grohmann U, Vacca C, Bianchi R, Orabona C, Spreca A et al. T cell apoptosis by tryptophan catabolism. Cell Death Differ 2002; 9: 1069-1077. 
63. Bronte V, Zanovello P. Regulation of immune responses by L-arginine metabolism. Nat Rev Immunol 2005; 5: 641-654.

64. Hibbs Jr JB, Taintor RR, Vavrin Z. Macrophage cytotoxicity: role for L-arginine deiminase and imino nitrogen oxidation to nitrite. Science 1987; 235: 473-476.

65. Morris Jr SM. Arginine metabolism: boundaries of our knowledge. J Nutr 2007; 137 (6 Suppl 2): 1602S-1609S

66. Hesse M, Modolell M, La Flamme AC, Schito M, Fuentes JM, Cheever AW et al. Differential regulation of nitric oxide synthase-2 and arginase-1 by type 1/type 2 cytokines in vivo: granulomatous pathology is shaped by the pattern of L-arginine metabolism. $J$ Immunol 2001; 167: 6533-6544.

67. Munder M, Eichmann K, Moran JM, Centeno F, Soler G, Modolell M. Th1/Th2-regulated expression of arginase isoforms in murine macrophages and dendritic cells. $J$ Immunol 1999; 163: 3771-3777.

68. Tanaka Y, Guhde G, Suter A, Eskelinen EL, Hartmann D, Lullmann-Rauch R et al. Accumulation of autophagic vacuoles and cardiomyopathy in LAMP-2-deficient mice. Nature 2000; 406: 902-906.

69. Rodriguez PC, Quiceno DG, Ochoa AC. -arginine availability regulates T-lymphocyte cellcycle progression. Blood 2007; 109: 1568-1573.

70. Talloczy Z, Jiang W, Virgin HWT, Leib DA, Scheuner D, Kaufman RJ et al. Regulation of starvation- and virus-induced autophagy by the elF2alpha kinase signaling pathway. Proc Natl Acad Sci USA 2002; 99: 190-195.

71. Kaech SM, Ahmed R. Immunology. CD8T cells remember with a little help. Science 2003 300: 263-265.

72. Guerder S, Matzinger P. A fail-safe mechanism for maintaining self-tolerance. J Exp Med 1992; 176: 553-564.

73. Kennedy R, Celis E. Thelper lymphocytes rescue CTL from activation-induced cell death. J Immunol 2006; 177: 2862-2872.

74. Whiteside TL. Tumor-induced death of immune cells: its mechanisms and consequences. Semin Cancer Biol 2002; 12: 43-50.

75. O'Connell J, Bennett MW, O'Sullivan GC, Collins JK, Shanahan F. The Fas counterattack: cancer as a site of immune privilege. Immunol Today 1999; 20: 46-52.

76. Gastman BR, Atarshi Y, Reichert TE, Saito T, Balkir L, Rabinowich H et al. Fas ligand is expressed on human squamous cell carcinomas of the head and neck, and it promotes apoptosis of T lymphocytes. Cancer Res 1999; 59: 5356-5364.

77. Dworacki G, Meidenbauer N, Kuss I, Hoffmann TK, Gooding W, Lotze M et al. Decreased zeta chain expression and apoptosis in CD3+ peripheral blood T lymphocytes of patients with melanoma. Clin Cancer Res 2001; 7 (3 Suppl): 947s-957s.

78. Tanaka M, Suda T, Yatomi T, Nakamura N, Nagata S. Lethal effect of recombinant human Fas ligand in mice pretreated with Propionibacterium acnes. J Immunol 1997; 158 2303-2309.

79. Aoki K, Kurooka M, Chen JJ, Petryniak J, Nabel EG, Nabel GJ. Extracellular matrix interacts with soluble CD95L: retention and enhancement of cytotoxicity. Nat Immunol 2001; 2: 333-337.

80. Lenardo MJ. Interleukin-2 programs mouse alpha beta T lymphocytes for apoptosis. Nature 1991; 353: 858-861.

81. Russell JH, White CL, Loh DY, Meleedy-Rey P. Receptor-stimulated death pathway is opened by antigen in mature T cells. Proc Natl Acad Sci USA 1991; 88 2151-2155.

82. Griffith TS, Brunner T, Fletcher SM, Green DR, Ferguson TA. Fas ligand-induced apoptosis as a mechanism of immune privilege. Science 1995; 270: 1189-1192.

83. Bellgrau D, Gold D, Selawry H, Moore J, Franzusoff A, Duke RC. A role for CD95 ligand in preventing graft rejection. Nature 1995; 377: 630-632.

84. Arai $\mathrm{H}$, Gordon D, Nabel EG, Nabel GJ. Gene transfer of Fas ligand induces tumor regression in vivo. Proc Natl Acad Sci USA 1997; 94: 13862-13867.

85. Seino K, Kayagaki N, Okumura K, Yagita H. Antitumor effect of locally produced CD95 ligand. Nat Med 1997; 3: 165-170.

86. Chen JJ, Sun Y, Nabel GJ. Regulation of the proinflammatory effects of Fas ligand (CD95L). Science 1998; 282: 1714-1717.

87. Borst J, Hendriks J, Xiao Y. CD27 and CD70 in T cell and B cell activation. Curr Opin Immunol 2005; 17: 275-281.
88. Tesselaar K, Arens R, van Schijndel GM, Baars PA, van der Valk MA, Borst J et al. Lethal $T$ cell immunodeficiency induced by chronic costimulation via CD27-CD70 interactions. Nat Immunol 2003; 4: 49-54.

89. Py B, Slomianny C, Auberger P, Petit PX, Benichou S. Siva-1 and an alternative splice form lacking the death domain, Siva-2, similarly induce apoptosis in T lymphocytes via a caspase-dependent mitochondrial pathway. J Immunol 2004; 172: 4008-4017.

90. Chahlavi A, Rayman P, Richmond AL, Biswas K, Zhang R, Vogelbaum $M$ et al. Glioblastomas induce T-lymphocyte death by two distinct pathways involving gangliosides and CD70. Cancer Res 2005; 65: 5428-5438.

91. Dong H, Strome SE, Salomao DR, Tamura H, Hirano F, Flies DB et al. Tumor-associated B7-H1 promotes T-cell apoptosis: a potential mechanism of immune evasion. Nat Med 2002; 8: 793-800

92. Thompson RH, Kwon ED. Significance of B7-H1 overexpression in kidney cancer. Clin Genitourin Cancer 2006; 5: 206-211.

93. Konishi J, Yamazaki K, Azuma M, Kinoshita I, Dosaka-Akita H, Nishimura M. B7-H1 expression on non-small cell lung cancer cells and its relationship with tumor-infiltrating lymphocytes and their PD-1 expression. Clin Cancer Res 2004; 10: 5094-5100.

94. Galvan M, Tsuboi S, Fukuda M, Baum LG. Expression of a specific glycosyltransferase enzyme regulates T cell death mediated by galectin-1. J Biol Chem 2000; 275: 1673016737.

95. Matarrese P, Tinari A, Mormone E, Bianco GA, Toscano MA, Ascione B et al. Galectin-1 sensitizes resting human $T$ lymphocytes to Fas (CD95)-mediated cell death via mitochondrial hyperpolarization, budding, and fission. J Biol Chem 2005; 280: 6969-6985.

96. Chiang WF, Liu SY, Fang LY, Lin CN, Wu MH, Chen YC et al. Overexpression of galectin1 at the tumor invasion front is associated with poor prognosis in early-stage oral squamous cell carcinoma. Oral Oncol 2007; 22: 22.

97. van den Brule FA, Waltregny D, Castronovo V. Increased expression of galectin-1 in carcinoma-associated stroma predicts poor outcome in prostate carcinoma patients. J Pathol 2001; 193: 80-87.

98. Le QT, Shi G, Cao H, Nelson DW, Wang Y, Chen EY et al. Galectin-1: a link between tumor hypoxia and tumor immune privilege. J Clin Oncol 2005; 23: 8932-8941.

99. Zaks TZ, Chappell DB, Rosenberg SA, Restifo NP. Fas-mediated suicide of tumorreactive $T$ cells following activation by specific tumor: selective rescue by caspase inhibition. J Immunol 1999: 162: 3273-3279.

100. Saff RR, Spanjaard ES, Hohlbaum AM, Marshak-Rothstein A. Activation-induced cell death limits effector function of CD4 tumor-specific T cells. J Immunol 2004; 172: 65986606.

101. Kaech SM, Wherry EJ, Ahmed R. Effector and memory T-cell differentiation: implications for vaccine development. Nat Rev Immunol 2002; 2: 251-262.

102. Haring JS, Harty JT. Aberrant contraction of antigen-specific CD4 T cells after infection in the absence of gamma interferon or its receptor. Infect Immun 2006; 74: 6252-6263.

103. Chu CQ, Wittmer S, Dalton DK. Failure to suppress the expansion of the activated CD4 T cell population in interferon gamma-deficient mice leads to exacerbation of experimental autoimmune encephalomyelitis. J Exp Med 2000; 192: 123-128.

104. Berner V, Liu H, Zhou Q, Alderson KL, Sun K, Weiss JM et al. IFN-gamma mediates CD4+ T-cell loss and impairs secondary antitumor responses after successful initial immunotherapy. Nat Med 2007; 13: 354-360.

105. Haring JS, Badovinac VP, Harty JT. Inflaming the CD8+ T cell response. Immunity 2006; 25: $19-29$.

106. Kaech SM, Tan JT, Wherry EJ, Konieczny BT, Surh CD, Ahmed R. Selective expression of the interleukin 7 receptor identifies effector CD8 T cells that give rise to long-lived memory cells. Nat Immunol 2003; 4: 1191-1198.

107. Lepisto AJ, Mckolanis JR, Finn OJ. Cancer Immunotherapy: Challenges and Opportunities. Elsevier Inc, Amsterdam, The Netherlands, 2007

108. Gilboa E. DC-based cancer vaccines. J Clin Invest 2007; 117: 1195-1203.

109. Morgan RA, Dudley ME, Wunderlich JR, Hughes MS, Yang JC, Sherry RM et al. Cancer regression in patients after transfer of genetically engineered lymphocytes. Science 2006; 314: 126-129.

110. Finn OJ. Immune response as a biomarker for cancer detection and a lot more. $N$ Engl $J$ Med 2005; 353: 1288-1290. 